\title{
Physical Topology Design for Survivable Routing of Logical Rings in WDM-Based Networks
}

\author{
Aradhana Narula-Tam, Member, IEEE, Eytan Modiano, Senior Member, IEEE, and \\ Andrew Brzezinski, Student Member, IEEE
}

\begin{abstract}
In a wavelength-division multiplexed (WDM)-based network, a single physical link failure may correspond to multiple logical link failures. As a result, two-connected logical topologies, such as rings routed on a WDM physical topology, may become disconnected after a single physical link failure. We consider the design of physical topologies that ensure logical rings can be embedded in a survivable manner. This is of particular interest in metropolitan area networks, where logical rings are in practice almost exclusively employed for providing protection against link failures. First, we develop necessary conditions for the physical topology to be able to embed all logical rings in a survivable manner. We then use these conditions to provide tight bounds on the number of physical links that an $N$-node physical topology must have in order to support all logical rings for different sizes $K$. We show that when $K \geq 4$ the physical topology must have at least $4 N / 3$ links, and that when $K \geq 6$ the physical topology must have at least $3 N / 2$ links. Subsequently, we generalize this bound for all $K>4$. When $K>N-2$, we show that the physical topology must have at least $2 N-4$ links. Finally, we design physical topologies that meet the above bounds for both $K=4$ and $K=N-2$. Specifically, our physical topology for embedding $(N-2)$-node rings has a dual hub structure and is able to embed all rings of size less than $N-1$ in a survivable manner. We also provide a simple extension to this topology that addresses rings of size $K=N-1$ and rings of size $K=N$ for $N$ odd. We observe that designing the physical topology for supporting all logical rings in a survivable manner does not use significantly more physical links than a design that only supports a small number of logical rings. Hence, our approach of designing physical topologies that can be used to embed all possible ring logical topologies does not lead to a significant overdesign of the physical topology.
\end{abstract}

Index Terms-Network design, network survivability, routing, topology design, wavelength-division multiplexing (WDM).

\section{INTRODUCTION}

$\mathbf{O}$ PTICAL wavelength-division multiplexing (WDM) networks are a powerful architecture for making efficient use of the high bandwidth offered by optical fiber networks, through the ability to support along each fiber link the simultaneous

Manuscript received September 1, 2003; revised December 1, 2003. The work of A. Narula-Tam was supported in part by the Defense Advanced Research Projects Agency under Air Force Contract \#F19628-00-C-0002. The work of E. Modiano and A. Brzezinski was supported in part by the Defense Advanced Research Projects Agency under Grant MDA972-02-1-0021. Opinions, interpretations, recommendations, and conclusions are those of the authors and are not necessarily endorsed by the United States Government.

A. Narula-Tam is with the Massachusetts Institute of Technology Lincoln Laboratory, Lexington, MA 02420-9108 USA (e-mail: arad@11.mit.edu).

E. Modiano and A. Brzezinski are with the Laboratory for Information and Decision Systems, Massachusetts Institute of Technology, Cambridge, MA 02139-4307 USA (e-mail: modiano@mit.edu; brzezin@ mit.edu).

Digital Object Identifier 10.1109/JSAC.2004.830463 transmission of data along independent wavelength channels. A WDM network consists of a set of nodes, physically interconnected by optical fiber (the physical topology), upon which a logical topology is overlaid by establishing lightpath interconnections between the nodes. Since multiple connections may rely on a particular physical fiber link, there is potential for a significant loss of bandwidth upon the failure of a physical link.

In order to mitigate the effect of this and other types of network failure, the topic of optical layer protection has sprung up as an important research field [1]-[8]. Much of the work in WDM network protection is focused on restoration mechanisms that restore all lightpaths in the event of a physical link failure. There are two notable restoration methods, link-based restoration and path-based restoration. Link-based restoration recovers the failed physical link, consequently recovering the associated failed lightpaths. A common approach for link-based restoration is optical loop-back protection [2], [3], [5], [6]. Pathbased restoration recovers each failed lightpath by finding an alternative end-to-end path for each lightpath [2], [3], [8].

Often, however, physical layer restoration mechanisms may be unnecessary in the event of a physical link failure, due to the existence of alternate paths for transmitting data at the electronic layer. Of concern in this case is that the failure of a single physical link may lead to the failure of multiple logical links, leaving the logical topology disconnected. Thus, under a general physical topology the goal is to route the lightpaths in such a way that no single physical link failure leaves the logical topology disconnected. In this paper, we focus on logical ring topologies, where this design goal implies that no two logical links of the ring can traverse the same physical link. Clearly, if one logical link fails, loop-back protection at the electronic layer ensures that data may continue to transmit between any two nodes. We refer to this problem as the survivable routing problem for logical rings. Logical ring topologies enjoy widespread acceptance and are commonly the protected topologies of choice in metropolitan area networks.

The problem of finding survivable routings of logical ring topologies on a given physical topology was treated in [9]-[12]. The authors in [9]-[11] consider the necessary conditions for supporting survivable rings; additionally, [11] and [12] show that the problem of determining a survivable ring on a physical topology is NP-complete. A similar problem was considered in [7], where heuristic algorithms were developed in order to minimize the number of source-destination pairs that become disconnected upon a physical link failure. The algorithm in [7] uses tabu search procedures to find disjoint alternate paths for all of the lightpaths. 


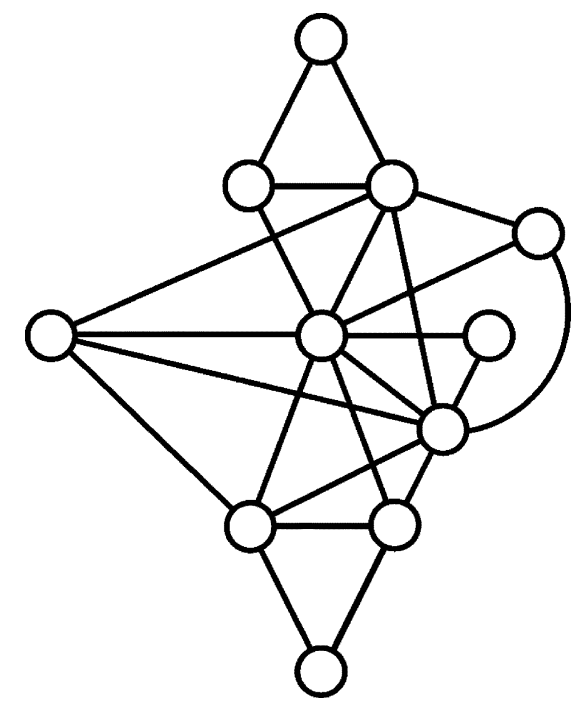

Fig. 1. New Jersey LATA.

The work in [7] and [10]-[12] considers the problem of finding a routing of the logical topology on a given physical topology, so that the ring remains connected in the event of a physical link failure. In contrast, this paper considers the problem of physical topology design. In particular, an important observation of [10] is that many physical topologies do not admit particular logical rings in a way that guarantees survivability. One example of this fact is that almost $44 \%$ of all nine-node rings cannot be embedded in a survivable manner in the 11-node NJLATA network shown in Fig. 1. Hence, in this paper, we seek to develop physical topology configurations that are amenable to supporting survivable logical rings. This approach is particularly applicable to greenfield network scenarios, where the flexibility exists to design the physical topology without the constraint of an existing infrastructure.

Such a design is particularly useful for service providers that design their network infrastructure in order to serve customer requests for lightpath connections. Alternatively, the service provider can provide restoration at the physical layer. However, such restoration may duplicate functionality provided at higher layers and be wasteful of resources. Also, the physical layer restoration must be fast enough to be compatible with the requirements of the higher layer (e.g., must restore the fiber cut before Synchronous Optical Network (SONET) initiates loop-back protection). Our approach to the physical topology design allows a service provider to route the lightpaths that constitute the logical ring along completely disjoint paths, thereby preserving the connectivity properties of the ring.

We consider the design of $N$-node physical topologies that can support survivable routings of logical ring topologies of size $K \leq N$. Clearly, rings of size 3 can be trivially embedded survivably in any two-connected physical topology, and as such our focus is on the problem of embedding rings of size $K \geq 4$. We begin in Section II with an analytical development of necessary conditions on the physical topology for ensuring all $K$-node ring permutations can be embedded in a survivable manner, for various values of $K$. These conditions take the form of lower bound requirements on the number of physical links in the net- work. Subsequently, in Section III, we formulate the problem as an integer linear program (ILP) to design the physical topology using the minimum number of physical links, while allowing a set of arbitrarily chosen logical topologies to be embedded in a survivable manner. In Section IV, we use the insights gained from Sections II and III to design physical topologies that can support all ring permutations in a survivable manner using the minimum number of physical links. Finally, Section $V$ provides a preliminary investigation of symmetric physical topologies for supporting logical rings.

\section{NeCESSARy CONDITIONS FOR SURVIVABLE ROUTING OF LOGICAL RINGS}

We consider a bidirectional physical topology with node set $\mathcal{N}$ and edge set $\mathcal{E}$ (we define $N \equiv|\mathcal{N}|$ as the number of nodes in the physical topology). Similarly, each bidirectional logical topology consists of a set of nodes $\mathcal{N}_{L}$ and edges $\mathcal{E}_{L}$. A cut is a partition of the set $\mathcal{N}$ into subsets $\mathcal{S}$ and $\mathcal{N} \backslash \mathcal{S}$. ${ }^{1}$ The cut-set corresponds to the set of edges in $\mathcal{E}$ that have one endpoint in $\mathcal{S}$ and the other in $\mathcal{N} \backslash \mathcal{S}$, and is denoted by $C S(\mathcal{S}, \mathcal{N} \backslash \mathcal{S})$.

In [10] and [11], necessary conditions on the physical topology were introduced to ensure survivable routing of ring logical topologies. We summarize the result in the following discussion and in Theorem 2.1, which is a crucial result upon which the remainder of the paper builds. Consider an arbitrary ring logical topology. For any cut $\{\mathcal{S}, \mathcal{N} \backslash \mathcal{S}\}$ of the physical topology, let $\left|C S_{P}(\mathcal{S}, \mathcal{N} \backslash \mathcal{S})\right|$ be the number of physical links along this cut and $\left|C S_{L}(\mathcal{S}, \mathcal{N} \backslash \mathcal{S})\right|$ be the number of logical links traversing the same cut. Clearly, in order to be able to route the logical links along disjoint physical paths, $\left|C S_{P}(\mathcal{S}, \mathcal{N} \backslash \mathcal{S})\right|$ must be greater than or equal to $\left|C S_{L}(\mathcal{S}, \mathcal{N} \backslash \mathcal{S})\right|$ for each cut. This condition is necessary, but not sufficient to ensure that a survivable routing exists for a particular ring logical topology. For embedding all possible $K$-node ring logical topologies in a survivable manner, the following necessary condition on the physical topology appeared in [10] and [11].

Theorem 2.1: For a physical topology to support any possible $K$-node ring logical topology in a survivable manner the following must hold. For any cut $\{\mathcal{S}, \mathcal{N} \backslash \mathcal{S}\}$ of the physical topology

$$
\left|C S_{P}(\mathcal{S}, \mathcal{N} \backslash \mathcal{S})\right| \geq 2 \min \left\{|\mathcal{S}|,|\mathcal{N} \backslash \mathcal{S}|,\left\lfloor\frac{K}{2}\right\rfloor\right\} .
$$

The proof of Theorem 2.1 is by construction: a logical ring topology can be created that alternates between nodes of the two sets $\mathcal{S}$ and $\mathcal{N} \backslash \mathcal{S}$ such that $2 \min \{|\mathcal{S}|,|\mathcal{N} \backslash \mathcal{S}|,\lfloor K / 2\rfloor\}$ physical links are in the cut-set (details may be found in [10], [11]). The theorem says that for all cuts of the physical topology, the number of physical links in the cut set must be greater than or equal to twice the minimum of the number of nodes on the smaller side of the cut and $\lfloor K / 2\rfloor$, where $\lfloor K / 2\rfloor$ corresponds to the maximum number of nodes in a $K$-node ring logical topology that can be on both sides of the cut. Note that Theorem 2.1 provides a necessary but not sufficient condition for a

${ }^{1}$ For sets $\mathcal{A}$ and $\mathcal{B}$, the set $\mathcal{A} \backslash \mathcal{B}$ is defined as $\mathcal{A} \cap \mathcal{B}^{C}$, where $\mathcal{B}^{C}$ is the complement of the set $\mathcal{B}$. 
TABLE I

LOWER BOUNDS ON THE NUMBER OF PHYSICAL LINKS REQUIRED TO EMBED LOGICAL TOPOLOGIES OF SIZE $K$

\begin{tabular}{c|c|c}
\hline \hline $\begin{array}{c}\text { Logical Ring } \\
\text { Size }\end{array}$ & $\begin{array}{c}\text { Physical Link } \\
\text { Requirement }\end{array}$ & Result \\
\hline$K=4$ & $4 N / 3$ & Theorem 2.2 \\
$K=6$ & $3 N / 2$ & Theorem 2.3 \\
$K=8$ & $1.6 N$ & Theorem 2.4 \\
$K=10$ & $1.625 N$ & Theorem 2.4 \\
$K=N-2$ & $2 N-4$ & Theorem 2.5 \\
\hline \hline
\end{tabular}

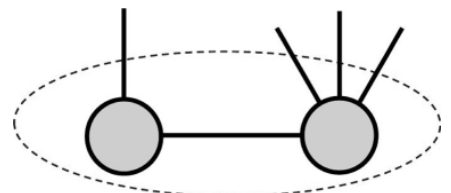

Fig. 2. Two-node cut-set consisting of a degree 2 node connected to a degree 4 node.

physical topology to support the survivable routing of all possible $K$-node ring logical topologies.

Using Theorem 2.1, we develop lower bounds on the number of physical links needed to embed rings of size $K$ for evenvalued $K \geq 4$. We also show in Theorem 2.5, that to embed rings of size $K=N-2$, a minimum of $2 N-4$ physical links are needed. A summary of several of the lower bound results derived in this section is given in Table I.

In order to establish the lower bound for the case $K \geq 4$, we take advantage of the following lemma, which makes use of Theorem 2.1 to show that a node of degree 2 cannot have a physical link to a node of degree 2 or 3 , and a node of degree 3 cannot have a physical link to a node of degree 2 .

Lemma 2.1: Any node of degree $D$ must have physical links to nodes having degree at least

$$
\begin{cases}4, & \text { for } D=2 \\ 3, & \text { for } D=3\end{cases}
$$

when $K \geq 4$ and $N \geq 4$.

Proof: We consider the two cases separately by looking at cuts of node pairs from the physical topology. Assume that $K \geq 4$ and $N \geq 4$. To prove the lemma, we consider two-node cuts, where one of the two nodes has degree $D$.

Consider first the case $D=2$. Theorem 2.1 requires that there are at least four edges crossing the cut (this follows since $|\mathcal{S}|=$ 2 implies $2 \min \{|\mathcal{S}|,|\mathcal{N} \backslash \mathcal{S}|,\lfloor K / 2\rfloor\}=4$ ). Since $D=2$, the other node in the cut must have degree at least 4 to satisfy this requirement. This is illustrated in Fig. 2: Note that if the node of degree 2 were connected instead to a node of degree 2 or 3 , then an insufficient number of links would cross the cut.

Next, consider the case where $D=3$. The same requirement of four edges crossing the cut holds here, which implies that the second node must have degree at least 3 .

Note that Lemma 2.1 provides no restriction on nodes of degree 4 or higher. Having established the supporting lemma, we begin our analysis of physical link requirements for embedding logical rings of size $K$, by considering first the case $K=4$. Our first result is summarized in the following theorem.

Theorem 2.2: To support all logical rings of size $K \geq 4$, an $N$-node physical topology must have at least $4 N / 3$ links.

Proof: Let $d_{i}$ be the number of nodes with degree $i$ in the physical topology. Then, the number of links $L$ in the physical topology is

$$
L=\sum_{i=2}^{N-1} \frac{i d_{i}}{2}=d_{2}+\frac{3 d_{3}}{2}+\sum_{i=4}^{N-1} \frac{i d_{i}}{2} .
$$

Recall from Lemma 2.1 that any node of degree 2 must have physical links to nodes of degree 4 or higher. Therefore, an upper bound on $d_{2}$ is

$$
2 d_{2} \leq \sum_{i=4}^{N-1} i d_{i} .
$$

Combining (1) with the inequality from (2), we obtain

$$
L \geq 2 d_{2}+\frac{3}{2} d_{3} .
$$

Since $\sum_{i=2}^{N-1} d_{i}=N$, (1) may be used to obtain the bound

$$
L \geq \frac{2 d_{2}+3 d_{3}+4\left(N-d_{2}-d_{3}\right)}{2}=2 N-d_{2}-\frac{1}{2} d_{3} .
$$

Combining (3) and (4), we require that the number of physical links must satisfy

$$
L \geq \max \left\{2 d_{2}+\frac{3}{2} d_{3}, 2 N-d_{2}-\frac{1}{2} d_{3}\right\} .
$$

We can determine the values of $d_{2} \geq 0$ and $d_{3} \geq 0$ that minimize the number of physical links required under the bound of (5). This minimum value occurs when there is equality between the two terms in (5). Equating these terms, we obtain

$$
d_{2}=\frac{2 N-2 d_{3}}{3} \text {. }
$$

Substituting this value of $d_{2}$ into (3) yields

$$
L \geq \frac{4 N}{3}+\frac{d_{3}}{3} \geq \frac{4 N}{3}
$$

as desired.

Next, we develop a lower bound on the number of physical links required to support rings of size $K=6$.

Theorem 2.3: To support all logical rings of size $K \geq 6$, and $N$-node physical topology must have at least $3 N / 2$ links.

Proof: Let $d_{i}$ be the number of nodes with degree $i$ in the physical topology. Then, the number of links $L$ in the physical topology is

$$
L=\sum_{i=2}^{N-1} \frac{i d_{i}}{2}=d_{2}+\frac{3 d_{3}}{2}+2 d_{4}+\frac{5 d_{5}}{2}+\sum_{i=6}^{N-1} \frac{i d_{i}}{2} .
$$

Recall from Lemma 2.1 that any node of degree 2 must have physical links to nodes of degree 4 or higher. Also, applying the result of Lemma A.1 (found in Appendix A) that at most 
one node of degree 2 can have a physical link to each node of degree 4 or degree 5 , an upper bound on $d_{2}$ is

$$
2 d_{2} \leq d_{4}+d_{5}+\sum_{i=6}^{N-1} i d_{i}
$$

Combining (7) with the inequality from (8), we obtain

$$
L \geq 2 d_{2}+\frac{3}{2} d_{3}+\frac{3}{2} d_{4}+2 d_{5}
$$

Since $\sum_{i=2}^{N-1} d_{i}=N,(7)$ may be used to obtain the bound

$$
\begin{aligned}
L & \geq \frac{1}{2}\left(2 d_{2}+3 d_{3}+4 d_{4}+5 d_{5}\right)+3\left(N-d_{2}-d_{3}-d_{4}-d_{5}\right) \\
& =3 N-2 d_{2}-\frac{3}{2} d_{3}-d_{4}-\frac{1}{2} d_{5} .
\end{aligned}
$$

Combining (9) and (11), we require that the number of physical links must satisfy

$$
\begin{aligned}
& L \geq \\
& \max \left\{2 d_{2}+\frac{3}{2} d_{3}+\frac{3}{2} d_{4}+2 d_{5}, 3 N-2 d_{2}-\frac{3}{2} d_{3}-d_{4}-\frac{1}{2} d_{5}\right\} .
\end{aligned}
$$

We can determine the values of $d_{2} \geq 0, d_{3} \geq 0, d_{4} \geq 0$, and $d_{5} \geq 0$ that minimize the number of physical links required under the bound of (12). This minimum value occurs when the two terms of (12) equate. Thus, we obtain

$$
2 d_{2}=\frac{3 N-3 d_{3}-\left(\frac{5}{2}\right) d_{4}-\left(\frac{5}{2}\right) d_{5}}{2} .
$$

Substituting this value of $d_{2}$ into (9) yields

$$
L \geq \frac{3 N}{2}+\frac{1}{4} d_{4}+\frac{3}{4} d_{5} \geq \frac{3 N}{2}
$$

as desired.

Using an approach similar to the proofs for Theorems 2.2 and 2.3 , we generalize the lower bound on $L$, the number of links in the physical topology, for arbitrary even values of $K$. This leads to the bounds for $K=8$ and 10 listed in Table I, and to lower bounds for $L$ that hold for all even $K \geq 8$, as long as $N$ is sufficiently large. The following theorem helps us to achieve this result.

Theorem 2.4: Suppose $K \geq 8$ is even. Let $d_{i}$ be the number of nodes with degree $i$ in the physical topology. To support all logical rings of size $K$, the number of physical links $L$ must satisfy (14), shown at the bottom of the page, for $N \geq K$.

Proof: The proof may be found in Appendix A.

It is clear that the property of supporting all logical rings exhibits a monotonicity property, in that a physical topology that supports the survivable routing of all logical rings of size $K$ also
TABLE II

LOWER BOUNDS IMPLIED BY THEOREMS 2.2, 2.3, AND 2.4 ON THE NUMBER OF PhysiCAl LiNKS REQUIRED TO EMBED LOGICAL TOPOLOGIES OF SizE $K$

\begin{tabular}{c|c}
\hline \hline $\begin{array}{c}\text { Logical Ring } \\
\text { Size }\end{array}$ & $\begin{array}{c}\text { Physical Link } \\
\text { Requirement }\end{array}$ \\
\hline$K=4,5$ & $4 N / 3$ \\
$K=6,7$ & $3 N / 2$ \\
$K=8,9$ & $1.6 N$ \\
$K=10,11, \ldots, 21$ & $1.625 N$ \\
\hline \hline
\end{tabular}

supports all logical rings of size less than $K$ survivably. Then, it is immediate that the lower bound for a particular value of $K$ also applies to all values greater than $K$. In particular, this means that our bounds may be extended to apply to odd values of $K$. For example, the bound for $K=4$ also may be applied as a bound for $K=5$ or greater.

Having established the lower bound on $L$ in Theorem 2.4, we may use (14) to find physical link requirements for particular values of $K$. Equation (14) becomes increasingly complex as $K$ increases. As such, a linear program may be employed to minimize over (14) and establish lower bounds for any $K \geq 8$. Table II summarizes the lower bounds on $L$ implied by Theorems $2.2,2.3$, and 2.4 for $K$ up to $K=21$. Of note is that the bound does not change when $K$ is increased above 10 .

Though the bound established in Theorem 2.4 appears to saturate for $K \geq 10$, we have not established that this bound is tight. In Section IV, we demonstrate a class of physical topologies that achieves the bound of $L=4 N / 3$ for $K=4$. However, no such topology has been found for higher values of $K$, when $K$ is of constant order relative to $N$. The simulation results of Section III suggest that higher values of $K$ are suited naturally by hub architectures that require on the order of $2 N$ physical links.

Having considered survivable routings of rings of size $K$ when $K$ is some constant, it becomes desirable to determine bounds on the case when $K$ is on the order of $N$. In particular, we consider the case of $K=N-2$.

Theorem 2.5: The minimum number of physical links necessary to support all logical rings of size greater than or equal to $N-2$ in a survivable manner is $2(N-2)$.

Proof: To prove Theorem 2.5, we show that for any physical topology with fewer than $2(N-2)$ links, we can find an $(N-2)$-node ring logical topology where each logical link requires two physical links (for a total of $2(N-2)$ links). Hence, a physical topology with fewer than $2(N-2)$ links cannot support all $(N-2)$-node logical topologies. The proof of Theorem 2.5 may be found in Appendix B.

In Section IV, we will provide a physical topology that achieves the bound of Theorem 2.5 for $K=N-2$ and another physical topology that is within one physical link of the same bound for $K=N-1$ and $K=N$.

$$
L \geq \max \left\{2 d_{2}+\frac{3}{2} d_{3}+\sum_{\substack{i=4 \\ i \text { even }}}^{K-1}\left(\frac{i}{4}+\frac{1}{2}\right) d_{i}+\sum_{\substack{i=4 \\ \text { iodd }}}^{K-1}\left(\frac{i}{4}+\frac{3}{4}\right) d_{i}, \quad 2 d_{2}+\frac{5}{2} d_{3}+\sum_{i=4}^{K / 2} d_{i}, \quad \frac{K N}{2}-\frac{1}{2} \sum_{i=2}^{K-1}(K-i) d_{i}\right\}
$$


The results of this section provide us with lower bounds on the number of physical links that the physical topology requires. They also give us some insights regarding the structure of the topology (for example, low degree nodes tend to be connected to high degree nodes). However, they do not directly provide us with a physical topology design. In order to obtain additional insight, we next apply integer linear programming techniques to design physical topologies. In Section IV, we will use these insights to design physical topologies that meet the above bounds.

\section{InTEGer Linear Programming Formulation}

In this section, we develop an integer linear program (ILP) formulation for designing physical topologies that can support large numbers of logical rings in a survivable manner. We consider the problem of finding a physical topology with a minimum number of physical links and the associated survivable routings for a batch of $R$ ring logical topologies with $K$ nodes each. We use [11, Th. 1] to determine a survivable routing for each of the $R$ rings, which ensures that each logical topology remains connected even in the event of a physical link failure. In order to route a logical link $(s, t)$ on the physical topology, a corresponding path on the physical topology must be found between nodes $s$ and $t$. Such a path consists of a set of physical links connecting nodes $s$ and $t$, as well as wavelengths along those links. Let $f_{i, j}^{s, t}=1$ if logical link $(s, t)$ is routed on physical link $(i, j)$, and 0 , otherwise.

Our linear programming algorithm starts with a fully connected physical topology and assigns a cost of 1 to each physical link that is used. The batch of $R$ rings is embedded simultaneously, and we assign $y_{i, j}=1$ if any lightpath uses physical link $(i, j)$. We can now formulate the physical topology design problem as the following ILP (ILP 1), with the objective of minimizing the total number of physical links used.

ILP 1: Linear program to find a physical topology for embedding $R$ survivable rings.

$$
\text { Minimize } \sum_{(i, j) \in \mathcal{E}} y_{i, j} \text { subject to }
$$

1) Connectivity constraints: For each pair $(s, t)$ in each logical $\operatorname{ring} \mathcal{E}_{L}$

$$
\sum_{\{j:(i, j) \in \mathcal{E}\}} f_{i, j}^{s, t}-\sum_{\{j:(j, i) \in \mathcal{E}\}} f_{j, i}^{s, t}= \begin{cases}1, & \text { if } s=i \\ -1, & \text { if } t=i \\ 0, & \text { otherwise }\end{cases}
$$

for all $i \in \mathcal{N}$.

2) Survivability constraints: For each logical ring $\mathcal{E}_{L}$

$$
\sum_{(s, t) \in \mathcal{E}_{L}} f_{i, j}^{s, t}+\sum_{(s, t) \in \mathcal{E}_{L}} f_{j, i}^{s, t} \leq 1
$$

for all $(i, j) \in \mathcal{E}$.

3) Physical link use constraints

$$
y_{i, j} \geq f_{i, j}^{s, t}
$$

for all $(i, j) \in \mathcal{E}$, all $(s, t) \in \mathcal{E}_{L}$, and all $\mathcal{E}_{L}$.

4) Integer flow constraints

$$
f_{i, j}^{s, t} \in\{0,1\} .
$$

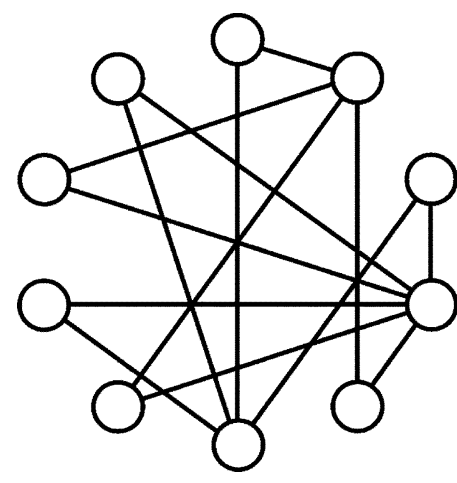

Fig. 3. Physical topology generated by ILP 1 for $K=6$ and $R=20$.

\section{A. Exact Solution for ILP I}

We implemented ILP 1 using the CPLEX software package. CPLEX uses branch and bound techniques for solving ILPs and is capable of solving ILPs consisting of up to one million variables and constraints. We have found that the solution of the ILP can only be determined for small problem instances. For example, with $N=10, K=6$, and $R=20$, a physical topology solution is found within 24 hours on a SPARC Ultra 10. The resulting physical topology is shown in Fig. 3. Due to the complexity of the linear programming solution, this approach cannot be used for general design. However, attributes of the resulting topologies found through experiments provide insights to the physical topology design problem. For example, we find that

1) physical topology does not contain a Hamiltonian cycle; 2) physical topology has a multihub structure.

In Section IV, we use these insights to design physical topologies that are capable of embedding all rings in a survivable manner. Our designs are consistent with the above observations and, in particular, we use an architecture based on two hubs.

\section{B. Heuristics for Solving ILP I}

We examined a number of approaches for solving ILP 1 with very little success. This included attempting to bundle the physical link constraints (item 3 in ILP 1) and attempting to relax the integer constraints. Another approach for obtaining a feasible (but not optimal) solution is to embed the rings sequentially rather than as a batch. Expectedly, designing physical topologies by sequentially embedding rings is not nearly as efficient as embedding the rings as a batch. For example, if we embed $N$-node rings on an $N$-node topology sequentially, the first set of links will be a Hamiltonian cycle that corresponds to the first logical ring. As mentioned above, the most efficient physical topologies may not contain a Hamiltonian cycle.

One approach that did prove rather successful was to embed a small batch of about 20 rings for which the above ILP can be solved and then use the resulting physical topology to sequentially embed additional rings, adding physical links when necessary. The intuition behind this approach is that by embedding the small batch, we avoid the negative effect that results from the sequential embedding of the first few rings. Moreover, it is reasonable to expect that the physical topology that results from embedding even a small batch is relatively close to the optimal topology for larger batches. For $N=9$, embedding a batch of 


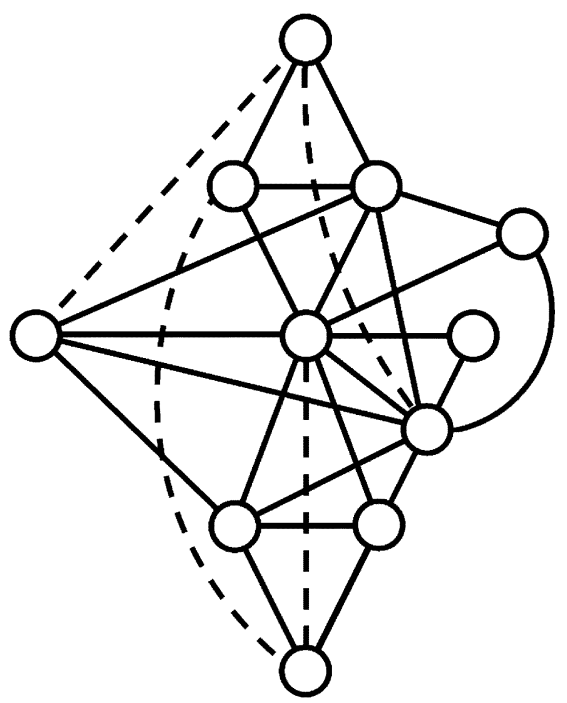

Fig. 4. New Jersey LATA with additional links added using our heuristic.

ten nine-node logical rings led to the modified dual hub architecture, described in Section IV and illustrated in Fig. 7. This architecture uses 15 links and can be shown to support all nine-node rings.

Another approach for solving the ILP is to express the objective function as a constraint. In other words, the objective function is removed and the constraint

$$
\sum_{(i, j) \in \mathcal{E}} y_{i j} \leq \text { MAXLINKS }
$$

is added. By varying the number of maximum physical links available, MAXLINKS, we can determine the minimum number of physical links required to embed the batch of logical rings. While this approach indeed reduced the running time of the ILP, we still only found it useful for relatively small problems. Hence, we combined the two approaches above, using the ILP to embed a small batch of rings with a maximum of MAXLINKS physical links to obtain an initial physical topology and then using this physical topology to embed more logical rings sequentially, adding physical links as required. Of course designing a physical topology in this manner to provably support all rings of size $N$ requires embedding $(N-1)$ ! rings, which is impractical for large $N$, even if the design is done offline. However, if we sequentially embed randomly selected rings, we find that the physical topology converges after embedding a relatively small number of rings. Thus, we can design physical topologies in this manner to have a high probability of being able to support all ring permutations.

This heuristic can also be used to improve existing physical topologies. For example, consider the NJLATA, which is capable of supporting only $56 \%$ of all nine-node rings. We embedded 100000 randomly selected nine-node rings sequentially, adding physical links when necessary. After embedding fewer than 1000 rings, the physical topology had converged and no additional links were needed to embed the remaining topologies. A total of four physical links were appended to the physical topology, as illustrated in Fig. 4.

We can also use this heuristic approach to create physical topologies with maximum nodal degree. This is useful in cases

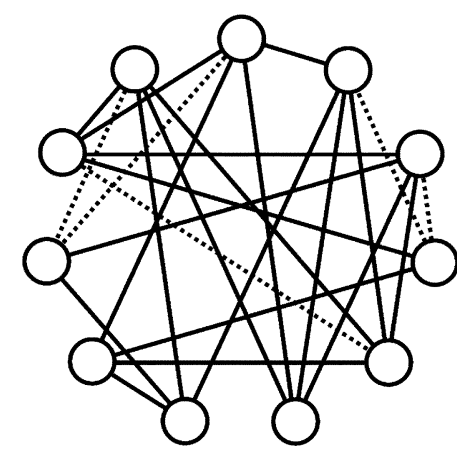

Fig. 5. Eleven-node topology with maximum nodal degree of 5 generated by the heuristic.

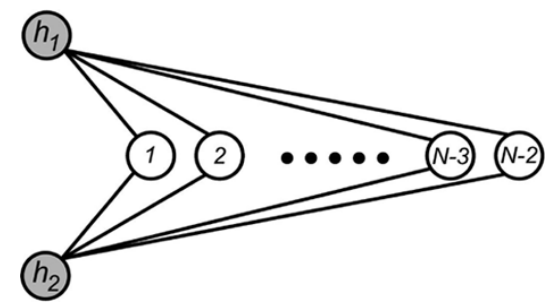

Fig. 6. Dual hub architecture.

where hub physical topologies are impractical. Other physical constraints could similarly be added. For example, a cost may be associated with each potential physical link, representing the relative cost of creating the physical connection. We embedded a batch of 20 nine-node rings on an 11-node physical topology using a maximum of 20 physical links and restricting the nodal degree to 5. We then embedded 100000 randomly selected nine-node rings. After less than 1000 ring embeddings, the physical topology had converged and is illustrated in Fig. 5, with the links that were added from sequentially embedding 100000 randomly selected nine-node rings marked as dashed lines. The resulting design requires 25 links rather than the 18 needed for the dual hub architecture, described in Section IV and illustrated in Fig. 6.

\section{Physical Topologies That EnSURE SURVIVABLE RING ROUTING}

The analytical and simulation (ILP) results provide valuable insights in designing physical topologies that can support ring permutations of various sizes. From Lemma 2.1, we know that all degree 2 nodes must have neighbors of degree 4 or higher. The physical topologies designed through the ILP simulations all have hub structures, i.e., a small number of nodes having high degrees and the remaining nodes with low degrees. We introduce several physical topologies that exhibit some of the key observations and results of the previous sections.

\section{A. Hub Architectures}

Dual Hub Architecture: Consider a physical topology with $N$ nodes, two of which are hub nodes. Each nonhub node has degree 2 and is connected to both hub nodes. The hub nodes each have degree $N-2$. Fig. 6 depicts the physical topology for a dual hub architecture having $N$ nodes. This physical topology 
has $2 N-4$ links, which is the lower bound established in Theorem 2.5 for routing all logical rings of size $K=N-2$. The following theorem establishes that when $N$ is even, any $(N-2)$-node ring logical topology may be routed using the dual hub architecture. It also demonstrates that for any $N$, the dual hub architecture supports all logical rings of size $K \leq N-3$.

Theorem 4.1: The dual hub architecture can support survivable routings of all logical rings of size $K=N-2$ for $N$ even and $K \leq N-3$ for $N$ odd.

Proof: The proof divides the possible logical ring configurations into three cases, depending on how many hubs are included in the logical ring. We defer the proof to Appendix $\mathrm{C}$ and provide three representative examples of the routing of $(N-$ 2 )-node rings on the dual hub architecture.

For the case of $N$ odd, it can be shown that the dual hub architecture is not sufficient for supporting all logical rings of size $N-2$. We now present some examples of routings of $(N-$ 2 )-node logical rings. Assume for each example that $N$ is even.

Example 4.1: Suppose we wish to route the logical ring defined by $(1,2, \ldots, N-2)$. That is, all nonhub nodes appear in order on the ring with no hub nodes included. Then, starting at node 1, the logical ring may be routed as follows: node 1 connects to node 2 through hub $h_{1}$, and node 2 connects to node 3 through hub $h_{2}$. We continue alternating between hub nodes in reaching the remaining nodes. Since $N$ is even, when we reach node $N-2$, we have reached it from hub $h_{1}$, which means we may complete the ring by traversing to node 1 through hub $h_{2}$.

Example 4.2: Suppose we wish to route the logical ring defined by $\left(h_{1}, 1,2, \ldots, N-3\right)$. Here, only hub $h_{1}$ appears on the ring, followed by nodes 1 through $N-3$ in order. Starting at hub $h_{1}$, the logical ring may be routed as follows: hub $h_{1}$ connects to node 1 directly, and node 1 connects to node 2 through hub $h_{2}$. Continuing to alternate between hub nodes in reaching the remaining nodes as before, we reach node $N-3$ from $h_{1}$. Since the direct link back to $h_{1}$ has been used, we route the last logical link from node $N-3$ through nodes $h_{2}$ and $N-2$ to hub $h_{1}$. $\square$

Example 4.3: Suppose we wish to route the logical ring defined by $\left(h_{1}, h_{2}, 1,2, \ldots, N-4\right)$. Here, the hubs are adjacent in the logical topology, followed by nodes 1 through $N-4$ in order. Starting at hub $h_{1}$, the logical ring may be routed as follows: hub $h_{1}$ connects to hub $h_{2}$ by traversing node $N-3$. Then, node 1 is reached directly from hub $h_{2}$ and the remaining nodes are reached in the alternating manner described in Examples 4.1 and 4.2. This implies that node $N-4$ is reached from hub $h_{1}$. Thus, the last logical link is routed from node $N-4$ through nodes $h_{2}$ and $N-2$ to hub $h_{1}$.

Having addressed the embedding of logical rings of size $N-2$ using the minimum number of links possible when $N$ is even, we now consider the case of $K=N-2$ for $N$ odd, as well as the cases of $K=N-1$ and $K=N$.

Modified Dual Hub Architecture: The addition of a single link directly joining the two hub nodes provides an architecture well suited to the cases of $K=N-2, K=N-1$, and $K=N$. We call this architecture the modified dual hub architecture and depict it in Fig. 7.

Theorem 4.2: The modified dual hub architecture with $N$ nodes supports all $(N-1)$-node logical rings in a survivable manner.

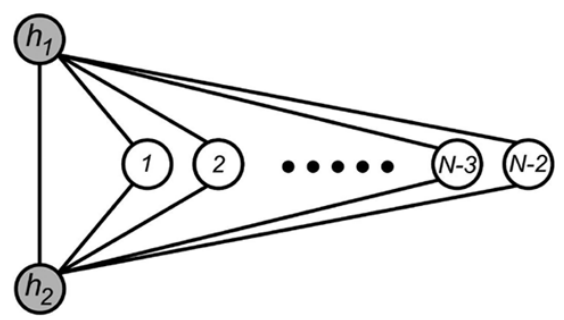

Fig. 7. Modified dual hub architecture.

Proof: The logical topology may have either one or two hub nodes. All possible logical ring topologies are fully described by the $(N-1)$-node analogues of configurations (37) and (38), which may be found in the proof of Theorem 4.1 in Appendix C. The routings for both configurations proceed similarly to the one- and two-hub node cases detailed in the proof of Theorem 4.1, and are omitted for brevity.

Theorem 4.3: The modified dual hub architecture with $N$ nodes supports all $N$-node logical rings in a survivable manner when $N$ is odd.

Proof: The logical topology includes all nodes in the physical topology, and may be described by the $N$-node analogue of configuration (38). Since we are assuming $N$ to be odd, then we are assured that an even number of nodes separate $h_{1}$ and $h_{2}$ in one direction (clockwise or counterclockwise) and, consequently, an odd number of nodes separate $h_{1}$ and $h_{2}$ in the opposite direction. With this fact, a similar routing to that described in the proof of Theorem 4.1 (see Appendix C) for configuration (38) guarantees the routing of $N$-node logical rings. We omit further details of the proof for brevity.

Of note is that in the case of an even number of nodes, if an odd number of nodes separate $h_{1}$ and $h_{2}$ in the clockwise direction and in the counterclockwise direction, then the dual hub architecture is sufficient to route any such logical ring of size $K=N$. In general, however, when $N$ is even, the modified dual hub architecture is not sufficient to route all rings of size $N$. It can be shown that adding a second physical link joining the hub nodes solves the problem of routing all logical rings of size $N$, for any $N$.

We have shown using hubbed architectures that the lower bounds established in Section II are tight for the cases of $K=$ $N-2, K=N-1$, and $K=N$.

\section{B. Embedding Four-Node Rings}

We now introduce the design of a physical topology for embedding rings of size $K=4$. The physical topology only has nodes of degree 2 and degree 4 : each node of degree 2 is connected to two nodes of degree 4 and each node of degree 4 is connected to four nodes of degree 2 . Thus, there are twice as many degree 2 nodes as degree 4 nodes. This design adheres to Lemma 2.1, which restricts degree 2 nodes to connect to nodes of degree 4 or higher. An example physical topology with 12 nodes is shown in Fig. 8. The $N$-node generalization of this topology is completely described as follows.

1) Node 1 has links to nodes $2,3, N-1$, and $N$.

2) Node $i=3 j+1$ has links to nodes $i-1, i-2, i+1$, $i+2$, for $j=1, \ldots,(N / 3)-1$. 


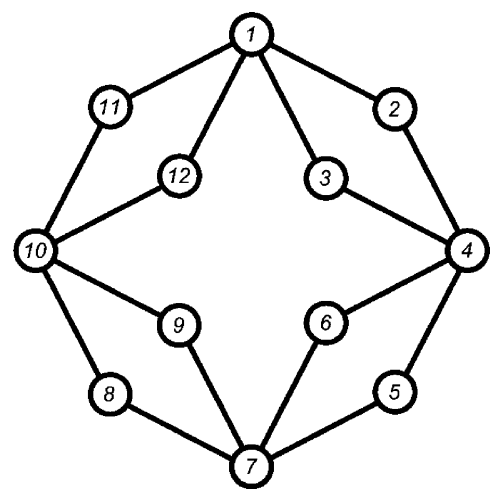

Fig. 8. Physical topology that can embed all rings of size 4 .

Clearly, the only allowed values of $N$ for which this topology may be constructed are given by $N=6+3 i$, for $i=0,1,2, \ldots$. We show below that the resulting physical topology can support all logical rings of size $K=4$. We then show that the physical topology consists of $4 N / 3$ physical links which is the minimum required to embed all four-node rings, as shown in Theorem 2.2.

Theorem 4.4: Consider an $N$-node physical topology consisting of only degree 2 and degree 4 nodes, interconnected as described above. This physical topology can support all rings of size $K=4$.

Proof: We consider a physical topology having $N$ nodes, numbered in a similar way to the topology shown in Fig. 8. Consider embedding an arbitrary logical ring consisting of four nodes in order, $(\pi(1), \pi(2), \pi(3), \pi(4))$. We will show that the physical topology can be divided into two connected circuits, ${ }^{2}$ one that contains nodes $\pi(1)$ and $\pi(2)$ and another that contains $\pi(3)$ and $\pi(4)$. Furthermore, the two circuits are connected at a degree 4 node, which we denote as $H$. Since any set of three nodes can be traversed in order on a circuit, the four lightpaths for the logical ring can be formed by traversing a path from $H$ to $\pi(1)$ to $\pi(2)$ to $H$ in the first circuit and then traversing a path from $H$ to $\pi(3)$ to $\pi(4)$ to $H$ in the second circuit.

Next, we address the existence of the circuits. The first circuit containing nodes $\pi(1)$ and $\pi(2)$ is formed by starting at node $\pi(1)$ and traversing the graph to reach node $\pi(2)$ and, subsequently, node $\pi(1)$. The constraint on this traversal is twofold: The circuit never traverses a physical link where the source node is numbered larger than the destination node, except when moving from either of nodes $N-1$ or $N$ to node 1 , and the circuit does not include the links adjacent to node $\pi(3)$ only if $\pi(3)$ has degree 2 [the same applies for node $\pi(4)$ ]. Note that any circuit satisfying these constraints is acceptable. Denote the set of edges traversed in this circuit by $\mathcal{E}_{\mathcal{M}}$. Then, the graph described by the original set of nodes $\mathcal{N}$ and the reduced set of edges $\mathcal{E} \backslash \mathcal{E}_{\mathcal{M}}$ must contain physical links that form a ring. Furthermore, nodes $\pi(3)$ and $\pi(4)$ must be on this ring, since the remaining links must travel through all degree 4 nodes, and we have purposely avoided traversing degree 2 nodes from the set $\{\pi(3), \pi(4)\}$.

Finally, we must prove the existence of node $H$. We have established that both circuits must traverse every node of degree 4

\footnotetext{
${ }^{2} \mathrm{~A}$ circuit is an alternating sequence of nodes and edges (physical links), such that the edges are distinct and the end vertices coincide.
}

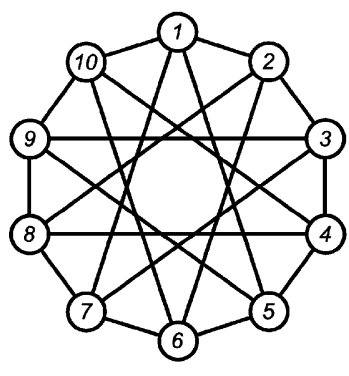

Fig. 9. Ten-node four-connected symmetric physical topology that supports all rings of size $K \leq 9$.

on the physical topology. Thus, without loss of generality, we take $H=1$.

Since the above topology contains twice as many degree 2 nodes as degree 4 nodes, clearly, it contains $4 N / 3$ physical links. Recall from Theorem 2.2 that this is the also the lower bound on the number of physical links required to support all rings of size 4 .

\section{SYMmetric PhysicAl TOPOLOGIES}

Designing physical topologies to embed survivable logical rings, while minimizing the number of physical links required led to the creation of physical topologies with multiple hubs. An additional property of these multiple hub topologies is that the physical topology is now also survivable to node failures. The physical network will always remain connected as long as one of the hub nodes is functioning. Hub physical topologies are generally easier to implement in local and metro area network environments. However, as the physical area of the network increases and due to other physical restrictions (such as right of ways, etc.) it may be impractical to deploy to multiple hubs. In this section, we present preliminary results on the design of physical topologies that are more symmetric, i.e., where the degree of each node is similar. This topic remains as an important area of future research.

\section{A. Rotationally Symmetric Topologies}

In [10], a ten-node four-connected symmetric physical topology (illustrated in Fig. 9) was shown that is capable of carrying all rings of size $K \leq 9$ in a survivable manner. This physical topology contains 20 physical links and each node has degree 4 . For comparison, the dual hub architecture would require 16 physical links in order to carry all logical rings of size $K \leq 9$ on a ten-node physical topology. Unfortunately, it is not possible to generalize this symmetric physical topology to all values of $N$ such that all rings of size $K \leq N-1$ can be routed survivably. The following theorem shows that this is so by addressing the survivable routing problem for a certain class of physical topologies. We begin by introducing the concept of a rotationally symmetric topology as a class of physical topologies containing the topology of Fig. 9.

Definition 5.1: A rotationally symmetric graph is a graph for which there exists a labeling of the $N$ vertices of the graph, 


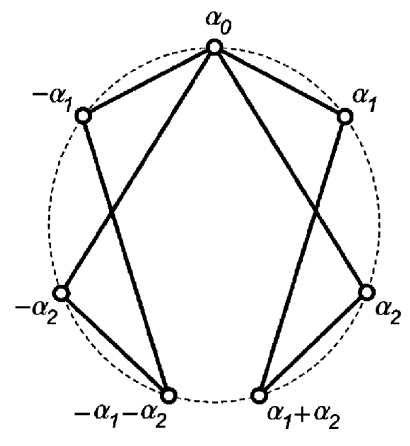

Fig. 10. Nodes in the cut $\mathcal{S}$ of Theorem 5.1.

denoted by vector $\left(v_{1}, \ldots, v_{N}\right)$, such that the incidence matrix ${ }^{3}$ of the graph is preserved if the vertices are relabeled with any of the $N-1$ circularly shifted versions of vector $\left(v_{1}, \ldots, v_{N}\right)$.

We refer to any physical topology that can be represented by a rotationally symmetric graph as a rotationally symmetric topology. Note that Fig. 9 depicts a rotationally symmetric topology, where each node has degree 4.

We will now consider any rotationally symmetric physical topology having $N$ nodes, each of degree 4 . In particular, since the topology is symmetric, we may pick an arbitrary node and give it the label $\alpha_{0}$. By Definition 5.1, the topology may be arranged in the shape of a ring with nodes appearing in the order $\left(v_{1}, \ldots, v_{N}\right)$. Since node $\alpha_{0}$ has degree 4 , it connects to a node $\alpha_{1}$ nodes away around the ring in the clockwise direction and to a second node $\alpha_{2}$ nodes away around the ring, also, in the clockwise direction (label these nodes $\alpha_{1}$ and $\alpha_{2}$, respectively). Note that without loss of generality, $\alpha_{1}<N / 2$ and $\alpha_{2}<N / 2$. By the symmetry of the topology, node $\alpha_{0}$ must connect to a node $\alpha_{1}$ nodes away in the counterclockwise direction, as well as a node $\alpha_{2}$ nodes away in the counterclockwise direction (label these nodes $-\alpha_{1}$ and $-\alpha_{2}$, respectively). From Fig. 9, if we label node 1 with $\alpha_{0}$, then $\alpha_{1}=2, \alpha_{2}=5,-\alpha_{1}=10$, and $-\alpha_{2}=7$.

Theorem 5.1: For $N \geq 14$, any rotationally symmetric topology where each node has degree 4 cannot support all logical rings of size $K \geq 14$.

Proof: Assume that $N \geq 14$ and $K \geq 14$. Labeling an arbitrary node as $\alpha_{0}$ as described above, consider a cut $\mathcal{S}$ containing the nodes $\alpha_{0}, \alpha_{1}, \alpha_{2},-\alpha_{1},-\alpha_{2}$, a node $\alpha_{1}+\alpha_{2}$ nodes in the clockwise direction from $\alpha_{0}$ (label this node as $\alpha_{1}+\alpha_{2}$ ), and a node $\alpha_{1}+\alpha_{2}$ nodes in the counterclockwise direction from $\alpha_{0}$ (label this node as $-\alpha_{1}-\alpha_{2}$ ). Note that this cut can possibly have size less than seven, since the node $\alpha_{1}+\alpha_{2}$ may overlap with either $-\alpha_{1},-\alpha_{2}$, or $-\alpha_{1}-\alpha_{2}$. We depict the nodes in the cut along with the edges that must interconnect these nodes in Fig. 10 when no overlap occurs between any of the nodes.

Since each node has degree 4, when no overlap occurs between any of the nodes, there are only 12 links in the cut-set. However, since $|\mathcal{S}|=7$, Theorem 2.1 requires that $12 \geq 2|\mathcal{S}|=$ 14 , which is impossible.

The remainder of the proof deals with cases where overlap occurs between the nodes of the cut. Suppose the node labeled

\footnotetext{
${ }^{3}$ The incidence matrix corresponding to a graph is an $N \times N$ matrix where the $(i, j)$ th element of the matrix is 1 if there exists an edge joining nodes $i$ and $j$ in the graph and 0 , otherwise.
}

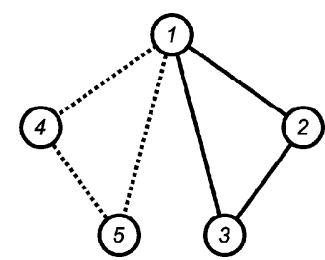

Fig. 11. Two interconnected Hamiltonian cycles can support all logical rings of size 5 in a survivable manner.

$\alpha_{1}+\alpha_{2}$ is also labeled $-\alpha_{1}$ (and consequently, the node labeled $-\alpha_{1}-\alpha_{2}$ is also labeled $\alpha_{1}$ ). Then, the cut $\mathcal{S}$, with $|\mathcal{S}|=5$, has four edges crossing the cut, but Theorem 2.1 requires that $4 \geq 2|\mathcal{S}|=10$, which is impossible. The same analysis applies to the case where the node labeled $\alpha_{1}+\alpha_{2}$ is also labeled $-\alpha_{2}$ (and consequently, the node labeled $-\alpha_{1}-\alpha_{2}$ is also labeled $\left.\alpha_{2}\right)$.

Finally, consider the case where the node labeled $\alpha_{1}+\alpha_{2}$ is also labeled $-\alpha_{1}-\alpha_{2}$ (this occurs in Fig. 9). Then, the cut $\mathcal{S}$, with $|\mathcal{S}|=6$, has eight edges crossing the cut, but Theorem 2.1 requires that $8 \geq 2|\mathcal{S}|=12$, which is impossible.

Note that Theorem 5.1 shows that we cannot form a rotationally symmetric topology with $2 N$ edges that is capable of routing all logical rings of size $K \geq 14$. In contrast, the hub topologies in Section IV require only $2 N-3$ edges to route all logical rings of size less than or equal to $N$, for all $N$.

\section{B. Interconnected Hamiltonian Cycles}

One method of generating physical topologies that are provably capable of supporting survivable rings is to select the physical links to form interconnected Hamiltonian cycles. For example, if the physical topology contains two interconnected Hamiltonian cycles, all rings of size $K \leq 5$ can be supported. One of the Hamiltonian cycles is used to connect the first three nodes and the second Hamiltonian cycle is used to connect the remaining two nodes in the logical topology. This is shown in Fig. 11, with only the nodes included in a five-node logical topology shown. The solid lines represent logical links mapped on the first Hamiltonian cycle, and the dashed lines represent logical links mapped on the second Hamiltonian cycle. This results in a four-connected physical topology which uses $2 N$ physical links. Comparing this design to our lower bounds on physical links required, we have shown using Theorem 2.3 that to embed all rings of size $K \geq 6$, a minimum of $3 N / 2$ physical links are required. Thus, the interconnected pair of Hamiltonian cycles may not be a very efficient design.

In general, designing a physical topology by interconnecting $M$ Hamiltonian cycles results in a $2 M$-connected physical topology that is capable of supporting rings of size $2 M+1$ in a survivable manner.

\section{CONCLUSION}

We have considered the problem of physical topology design for embedding logical rings in a survivable manner. This problem is particularly important for service providers that design their fiber infrastructure in order to support future customer requests for lightpath connections. Since rings are a very commonly used logical topology (due to their ability to recover from 
failures), we focused in this paper on design for ring logical topologies. Of course, a natural extension of this work is general design for arbitrary (two-connected) logical topologies.

We obtained some basic necessary conditions on the physical topology in order to be able to route logical rings in a survivable manner. We also developed lower bounds on the number of links that the physical topology must contain in order to be able to support all possible logical links of size $K$ (for various values of $K$ ). We explored a linear programming approach to the design of physical topologies. This approach pointed to hub architectures as being particularly well suited to the design problem. We designed a number of basic physical topologies to suit these bounds: for the cases of $K=4$ and $K=N-2$, the lower bounds are met exactly by the physical topologies introduced (the topology for embedding four-node rings and the dual hub architecture, respectively), while for the cases of $K=N-1$ and $K=N$, we have shown that the bound is tight using the physical topology introduced (the modified dual hub architecture). Since hub architectures may be impractical, we provided preliminary results relating to topologies where each node has equal degree. An important area of future research lies in exploring further designs that do not make use of hub nodes.

Finally, one may question our desire to support all $K$-node logical rings in a survivable manner. The question arises of whether we are overprovisioning the physical network in our quest to support all logical rings. Notice however, that our designs use fewer than $2 N$ physical links to support all logical rings of size $N-1$ or smaller (and in a large class of cases, of size $N$ as well). An arbitrary $(N-1)$-node logical topology requires a minimum of $N-1$ physical links in order to be two-connected. Furthermore, our experiments show that attempting to embed just a small number of logical rings already requires very close to $2 N$ physical links; hence, requiring the design to support all possible logical rings in fact does not result in a significant number of additional physical links. Moreover, the ability to support all logical rings is useful because it allows the logical ring topology to be reconfigured. Such reconfiguration has been shown to reduce network traffic loads [13], [14].

\section{APPENDIX A}

\section{PROOF OF THEOREM 2.4}

The proof of Theorem 2.4 will proceed by proving that each individual term from (14) serves as a lower bound on $L$. The first and last terms of (14) are obtained very similarly to the two terms that define the lower bound when $K=4$ and $K=6$ (see the proofs of Theorems 2.2 and 2.3). In order to obtain the first and second terms in (14), we introduce a new lemma that restricts the interconnections allowed between groups of nodes. Following the lemma, we provide three clarifying examples.

Definition A.1: Define a grouping as a set of $k_{2}+k_{3}$ nodes, $k_{2}$ of which have degree 2 and $k_{3}$ of which have degree 3 . Further, these nodes may be interconnected, but each node must have at least one single link free to connect to nodes outside of the grouping. For consistency in naming, we define the degree of this object by the pair $\left(k_{2}, k_{3}\right)$.
Lemma A.1: Suppose a node of degree $D$ connects to $n$ groupings, each of degree $\left(k_{2}, k_{3}\right)$, in the sense that the node of degree $D$ has a physical link to every node in each grouping. For $K$ and $N$ sufficiently large, an upper bound on $n$ is given by

$$
n \leq\left\lfloor\frac{D-2}{2 k_{2}+k_{3}}\right\rfloor
$$

Proof: The total number of nodes in a cut including $n$ groupings and the node of degree $D$ is $|\mathcal{S}|=n\left(k_{2}+k_{3}\right)+1$. Assume $|\mathcal{S}|$ achieves the minimum value in the set $\{|\mathcal{S}|,|\mathcal{N} \backslash \mathcal{S}|,\lfloor K / 2\rfloor\}$. Then, Theorem 2.1 requires that

$$
\begin{aligned}
2\left(1+n k_{2}+n k_{3}\right) & \leq\left|C S_{P}(\mathcal{S}, \mathcal{N} \backslash \mathcal{S})\right| \\
& \leq\left(n k_{2}+2 n k_{3}\right)+\left(D-n k_{2}-n k_{3}\right) .
\end{aligned}
$$

Here, the right-hand side of (17) is obtained by considering the maximum number of edges crossing the cut. This value occurs when all edges, excluding the edges connecting the node of degree $D$ to the nodes of the groupings, cross the cut. Thus, the node of degree $D$ contributes $D-n\left(k_{2}+k_{3}\right)$ edges that cross the cut [this is the first term in (17)]. The $n$ groupings contribute an additional $n\left(k_{2}+2 k_{3}\right)$ edges that cross the cut, since nodes of degree 2 contribute one edge each, and nodes of degree 3 contribute 2 edges each [this is the second term in (17)]. Simple algebraic manipulation of (17) yields

$$
n \leq \frac{D-2}{2 k_{2}+k_{3}} .
$$

Since $n$ is an integer, the bound in (15) is established.

The bounds on $K$ and $N$ that must be satisfied for this lemma to hold are as follows:

$$
\begin{aligned}
& K \geq \begin{cases}2\left(\left\lfloor\frac{D-2}{2 k_{2}+k_{3}}\right\rfloor\left(k_{2}+k_{3}\right)+1+k_{2}+k_{3}\right), & \text { for } K \text { even } \\
2\left(\left\lfloor\frac{D-2}{2 k_{2}+k_{3}}\right\rfloor\left(k_{2}+k_{3}\right)+\frac{3}{2}+k_{2}+k_{3}\right), & \text { for } K \text { odd }\end{cases} \\
& N \geq 2\left(\left\lfloor\frac{D-2}{2 k_{2}+k_{3}}\right\rfloor\left(k_{2}+k_{3}\right)+1+k_{2}+k_{3}\right) .
\end{aligned}
$$

We omit the details of the proofs of these bounds, only mentioning that they are based on the requirement that $K$ and $N$ are sufficiently large to test for and to find the upper bound on $n$, while maintaining $|\mathcal{S}|$ as the minimizing element of the set $\{|\mathcal{S}|,|\mathcal{N} \backslash \mathcal{S}|,\lfloor K / 2\rfloor\}$.

We will now provide three examples that demonstrate the usefulness and flexibility of Lemma A.1. The first two examples consider the very important cases of groupings of degree $(1,0)$ and $(0,1)$. [Lemma A.1 applied to a grouping of degree $(1,0)$ for the cases of $D=4,5$ was used in the proof of Theorem 2.3.] The third example serves to clarify the notion of a grouping. The examples all assume that $K$ and $N$ are sufficiently large for Lemma A.1 to apply.

Example A.1: Consider a grouping of degree $(1,0)$. For a node of degree $D=6$, we will use Lemma A.1 to determine how many of these groupings the degree 6 node may connect 


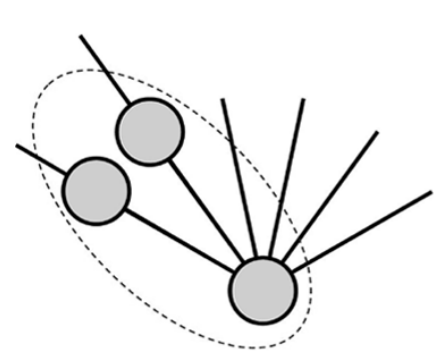

(a) two degree 2 nodes

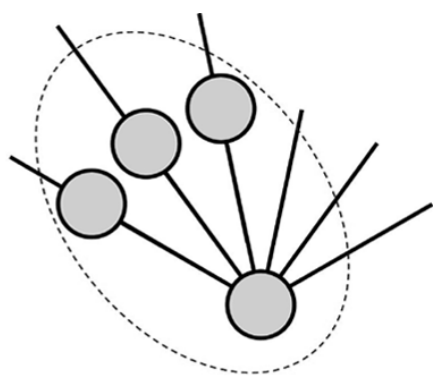

(b) three degree 2 nodes
Fig. 12. Node of degree 6 connecting to different numbers of nodes of degree 2.

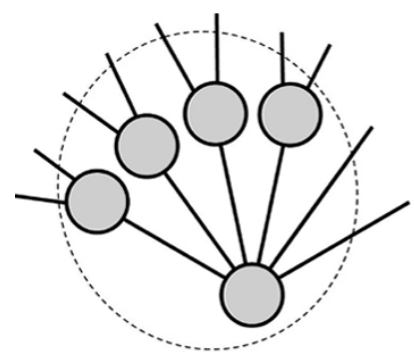

(a) four degree 3 nodes

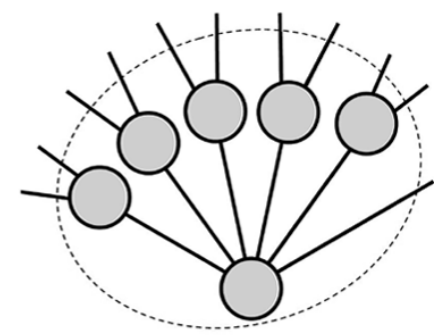

(b) five degree 3 nodes
Fig. 13. Node of degree 6 connecting to different numbers of nodes of degree 3 .

to without violating Theorem 2.1. In other words, we will determine the maximum number of nodes of degree 2 a degree 6 node may connect to. By Lemma A.1, this value is $\lfloor 4 / 2\rfloor=2$. We demonstrate this bound in Fig. 12: Fig. 12(a) shows that when the degree 6 node connects to two nodes of degree 2 , the minimum requirement of six edges may cross the cut. Fig. 12(b) adds an additional node of degree 2, which leaves a maximum of six edges crossing the cut (as shown). However, Theorem 2.1 requires a minimum of eight edges crossing a cut of four nodes. Thus, we have shown that a degree 6 node may connect to at most two nodes of degree 2.

Example A.2: Consider a grouping of degree $(0,1)$. In this case, Lemma A.1 sets a limit of $\lfloor 4 / 1\rfloor=4$ nodes of degree 3 that a node of degree $D=6$ may connect to. This is illustrated in Fig. 13: Fig. 13(a) shows that when the degree 6 node connects to four nodes of degree 3 , the minimum requirement of ten edges may cross the cut. Fig. 13(b) adds a fifth node of degree 3 to the cut, which leaves a maximum of 11 edges crossing the cut (as shown). However, Theorem 2.1 requires a minimum of 12 edges crossing a cut of six nodes. Thus, we have shown that a node of degree 6 may connect to at most four nodes of degree 3 .

Example A.3: The last example clarifies the notion of a grouping. Consider a grouping of degree $(1,1)$. In this case, Lemma A.1 sets a limit of $\lfloor 4 / 3\rfloor=1$ grouping of degree $(1,1)$ that a node of degree $D=6$ may connect to. This is illustrated in Fig. 14, where each grouping is shaded by a box: Fig. 14(a) shows that when the degree 6 node connects to one grouping of degree $(1,1)$, as many as seven edges may cross the cut. Fig. 14(b) shows that when the degree 6 node connects to two

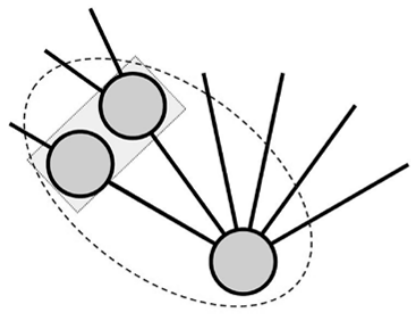

(a) one grouping having degree $(1,1)$

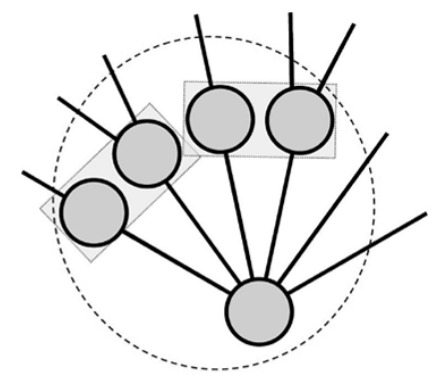

(b) two groupings, each having degree $(1,1)$
Fig. 14. Node of degree 6 connecting to different numbers of groupings of degree $(1,1)$.

groupings of degree $(1,1)$, a maximum of eight edges cross the cut. However, Theorem 2.1 requires a minimum of ten edges crossing a cut of five nodes.

Proof of Theorem 2.4: We begin by rewriting the expression for number of links in the physical topology $L$ as

$$
L=\sum_{i=1}^{K-1} \frac{i d_{i}}{2}+\sum_{i=K}^{N-1} \frac{i d_{i}}{2}
$$

Combining the fact (from Lemma 2.1) that nodes of degree 2 must have physical links to nodes of degree 4 or higher, with the bound of Lemma A.1 for groupings of degree $(1,0)$, which restricts the number of connections a node of degree $D$ can have to degree 2 nodes, we obtain the following restriction on the number of nodes of degree 2 :

$$
\begin{aligned}
2 d_{2} \leq\left(d_{4}+d_{5}\right) & +2\left(d_{6}+d_{7}\right)+3\left(d_{8}+d_{9}\right)+\cdots \\
& +\left(\frac{K}{2}-2\right)\left(d_{K-2}+d_{K-1}\right)+\sum_{i=K}^{N-1} i d_{i}
\end{aligned}
$$

Equation (20) may be used to eliminate the final term of (21), which provides the first restriction on $L$

$$
L \geq 2 d_{2}+\frac{3}{2} d_{3}+\sum_{\substack{i=4 \\ i \text { even }}}^{K-1}\left(\frac{i}{4}+\frac{1}{2}\right) d_{i}+\sum_{\substack{i=4 \\ \text { iodd }}}^{K-1}\left(\frac{i}{4}+\frac{3}{4}\right) d_{i}
$$

To achieve this bound, we applied Lemma 2.1, which requires $N \geq 4$, and Lemma A.1 for $D \in\{1,2, \ldots, K-1\}$. Using (18) and (19) for a grouping of degree $(1,0)$ and $K$ even, we find that (22) holds when $N \geq K$.

Next, we establish an upper bound on the value of $2 d_{2}+3 d_{3}$. From Lemma A.1, we have that a degree $D$ node connects to at most

$$
\left\lfloor\frac{D-2}{2 k_{2}+k_{3}}\right\rfloor\left(k_{2}+k_{3}\right)
$$

nodes, when these nodes necessarily belong to groupings of degree $\left(k_{2}, k_{3}\right)$. Note that $\left(k_{2}, k_{3}\right)=(0,1)$ maximizes $(23)$ over all possible groupings. To prove this, suppose that $\left(k_{2}^{*}, k_{3}^{*}\right)$ achieves the maximum in (23). If $k_{2}^{*}>0$, then note that $\left(0, k_{2}^{*}+\right.$ 
$\left.k_{3}^{*}\right)$ achieves a higher value by decreasing the denominator term, while having no effect on the numerator terms of (23). Next, suppose $k_{3}^{*}>1$ and $k_{2}^{*}=0$. Then, we have immediately

$$
\left\lfloor\frac{D-2}{k_{3}^{*}}\right\rfloor k_{3}^{*} \leq D-2 .
$$

Of course, this inequality is satisfied with equality when $k_{3}^{*}=1$. Then, the maximum number of nodes of degree 2 or 3 that a node of degree $D$ can reach is given by $D-2$. This implies that the following bound holds:

$$
\begin{aligned}
2 d_{2}+3 d_{3} \leq d_{3}+2 d_{4} & +3 d_{5}+\cdots \\
& +\left(\frac{K}{2}-2\right) d_{K / 2}+\sum_{K / 2+1}^{N-1} i d_{i} .
\end{aligned}
$$

Applying (24) to (20), we obtain the second bound on $L$

$$
L \geq 2 d_{2}+\frac{5}{2} d_{3}+d_{4}+d_{5}+\cdots+d_{K / 2}
$$

for $K \geq 8$. To achieve the bound of (25), we employed Lemma A.1. Since (24) is derived based on a grouping of degree $(0,1)$ and the assumption that $K$ is even, the bounds on $K$ and $N$ require that $N \geq K$.

Finally, the third bound on $L$ is obtained as follows. We lower bound the second term of (20) as

$$
\sum_{i=K}^{N-1} \frac{i d_{i}}{2} \geq K \sum_{i=K}^{N-1} \frac{d_{i}}{2}
$$

Using (20) and combining the fact that $\sum_{i=1}^{N-1} d_{i}=N$ with (26) provides the lower bound

$$
\begin{aligned}
L & \geq \frac{1}{2} \sum_{i=2}^{K-1} i d_{i}+\frac{K}{2}\left(N-\sum_{i=2}^{K-1} d_{i}\right) \\
& =\frac{K N}{2}-\frac{1}{2} \sum_{i=2}^{K-1}(K-i) d_{i} .
\end{aligned}
$$

The bounds of (22), (25), and (28) in combination correspond to the desired bound (14), which holds for all $N \geq K$, as desired.

\section{APPENDIX B \\ PROOF OF THEOREM 2.5}

The proof of Theorem 2.5 requires two supporting lemmas.

Lemma B.1: Given a graph with $N$ nodes with degrees $e_{1} \leq$ $e_{2} \leq \cdots \leq e_{N}$, if $e_{j}>j$ for $1 \leq j \leq m / 2$, the graph contains a Hamiltonian cycle.

Proof: The proof may be found in [15, p. 350].

Lemma B.2: Consider an $N$-node physical topology with $L \leq 2 N-5$ physical links. Let $d_{i}$ be the degree of the $i$ th largest degree node in the $N$-node topology and assume each node has a minimum degree of two. Then

$$
d_{i} \leq \frac{2 N-10+2 i}{i} .
$$

Proof: Since there are at most $2 N-5$ physical links in the physical topology, the sum of the degrees of all the nodes must be less than or equal to $4 N-10$. Equivalently

$$
\left(\sum_{k=1}^{i-1} d_{k}\right)+d_{i}+\left(\sum_{k=i+1}^{N-1} d_{k}\right) \leq 4 N-10 .
$$

Since the degree of each node is at least two, we clearly have that

$$
\sum_{k+1}^{N-1} d_{k} \geq 2(N-i)
$$

Combining (30) and (31), we obtain an upper bound on $d_{i}$

$$
d_{i} \leq 4 N-10-2(N-i)-\sum_{k=1}^{i-1} d_{k}=2 N-10+2 i-\sum_{k=1}^{i-1} d_{k} .
$$

Since $d_{i}$ is defined as the degree of the $i$ th largest degree node, its degree must be less than the average of the of the larger degree nodes

$$
d_{i} \leq \frac{1}{i-1} \sum_{k=1}^{i-1} d_{k} .
$$

Together, (32) and (33) provide

$$
d_{i} \leq \frac{2 N-10+2 i}{i}
$$

as desired.

Proof of Theorem 2.5: The proof is by construction of an $(N-2)$-node logical topology that requires at least $2 N-4$ physical links. The proof is divided into four cases corresponding to physical topology size. In the first case, we show that the theorem holds for all rings of size greater than or equal to 12 . The other three cases establish the proof for rings of size less than 12.

In Case 1, we consider physical topologies of size $N \geq 12$. We start by removing the two largest degree nodes from the physical topology. The resulting physical topology $\mathcal{P}$, has $m=$ $N-2$ nodes, with degrees $d_{3}$ through $d_{N}$. We show below that there exists a logical ring topology that traverses these $N-2$ nodes and requires more than $2 N-5$ physical links.

Consider the inverse of the $m$-node physical topology, denoted $\overline{\mathcal{P}}$, where link $(i, j) \in \mathcal{P}$ if $(i, j) \notin \overline{\mathcal{P}}$ and link $(i, j) \in \overline{\mathcal{P}}$ if $(i, j) \notin \mathcal{P}$. We will demonstrate that there exists a Hamiltonian cycle in this inverse graph. The existence of a Hamiltonian cycle in the inverse graph implies that there exists a sequence of $N-2$ nodes with direct physical links connecting them in the inverse topology. Hence, these $N-2$ nodes cannot be connected using direct physical links in the original physical topology, which implies that each logical link must utilize at least two physical links in the original physical topology. Thus, the logical ring corresponding to the Hamiltonian cycle in the inverse graph requires a minimum of $2 N-4$ physical links in the original topology.

We now proceed to prove our claim that a Hamiltonian cycle exists in the inverse topology. Let $i$ be the index of the $i$ th largest 
degree node in the original topology and let $\bar{d}_{i}$ denote its degree in the inverse graph $\overline{\mathcal{P}}$. Since $\overline{\mathcal{P}}$ has $N-2$ nodes, it must be true that $\bar{d}_{i} \geq N-3-d_{i}$. If the $i$ th largest degree node in the original topology has connections to nodes $i=1$ and $i=2$, then the degree of node $\bar{d}_{i}$ is strictly greater than $N-3-d_{i}$. Thus, $\bar{d}_{i+1}$ can be less than $\bar{d}_{i}$. However, the degree of the smallest degree node in $\overline{\mathcal{P}}$ is always greater than or equal to $N-3-d_{3}$ and the degree of the next smallest degree node in $\overline{\mathcal{P}}$ is always greater than or equal to $N-3-d_{4}$, and so on. To summarize, if $e_{j}$ denotes the degree of the $j$ th smallest degree node in $\overline{\mathcal{P}}$, then $e_{j} \geq N-3-d_{j+2}$.

Next, we apply Lemma B. 1 to the inverse graph $\overline{\mathcal{P}}$ to conclude that $\overline{\mathcal{P}}$ contains a Hamiltonian cycle if the degree of the nodes in the inverse graph are such that $e_{j}>j$ for $1 \leq j<m / 2$. Rewriting this requirement for a Hamiltonian cycle in terms of $d_{i}$ and $N$ yields

$$
e_{i-2} \geq N-3-d_{i}>i-2, \quad \text { for } 3 \leq i<\frac{N}{2}+1 .
$$

Using (29) from Lemma B.2 as an upper bound on $d_{i}$ in (34), we obtain the condition

$$
(N-i-1) i-2 N+10>0, \quad \text { for } 3 \leq i<\frac{N}{2}+1 .
$$

The roots of the left-hand side of (35) occur at $i=2$ and at $i=N-5$. It is easy to verify that (35) is true if $2<i<N-5$. Thus, (34) is satisfied if $N / 2+1 \leq N-5$. Consequently, there exists a Hamiltonian cycle in the inverse graph $\overline{\mathcal{P}}$ for all $N \geq$ 12. The resulting Hamiltonian cycle corresponds to a logical topology that requires for each logical link a minimum of two physical links in the original physical topology, i.e., this logical topology cannot be embedded in the original physical topology with fewer than $2 N-4$ physical links.

For Case 2, we consider physical topologies of size $N=6$ and $N=7$. Recall from Theorem 2.2 that a minimum of $4 N / 3$ physical links are required to support rings of size 4 or greater. Since $2 N-5<4 N / 3$ for $N \leq 7$, and Theorem 2.2 is valid for $N \geq 4$, Theorem 2.5 holds for $N=6$ and $N=7$.

Similarly, Case 3 corresponds to physical topologies of size $N=8$ and $N=9$. From Theorem 2.3, a minimum of $3 N / 2$ physical links are needed to support rings of size 6 or greater. Again, $2 N-5<3 N / 2$ for $N \leq 9$, and the result of Theorem 2.3 if valid for $N \geq 6$, which implies that Theorem 2.5 holds for $N=8$ and $N=9$.

Finally, Case 4 corresponds to physical topologies of size $N=10$ and $N=11$. From Theorem 2.4 a minimum of $1.6 N$ physical links are required to support rings of size 8 or greater. Since $2 N-5<1.6 N$ for $N \leq 12$, and the result of Theorem 2.4 for $K=8$ requires that $N \geq 8$, Theorem 2.5 holds.

\section{APPENDIX C \\ PROOF OF THEOREM 4.1}

We consider the case of $N$ even, and demonstrate the routing of any logical ring of size $K=N-2$. The proof for the case of $K=N-3$ for any $N$ has a similar progression and is omitted for brevity.

To prove that this physical topology can support all rings of size $N-2$, we need to show that each of the $(N-3)$ ! possible ring configurations can be routed on the dual hub architecture. Any logical ring configuration may be denoted by the vector $(\pi(1), \pi(2), \ldots, \pi(N-2))$, where $\pi(i)$ is the $i$ th node on the logical ring. We divide the possible configurations into three cases, where the logical ring contains 0,1 , or 2 of the hub nodes. Then, the logical ring may be expressed as

$$
\begin{gathered}
(1, \pi(2), \pi(3), \ldots, \pi(N-2)), \text { for } 0 \text { hub nodes } \\
\left(h_{1}, \pi(2), \pi(3), \ldots, \pi(N-2)\right), \text { for } 1 \text { hub node }
\end{gathered}
$$

or one of

$$
\left\{\begin{array}{c}
\left(h_{1}, h_{2}, \pi(3), \pi(4), \ldots, \pi(N-2)\right) \\
\left(h_{1}, \pi(2), h_{2}, \pi(4), \ldots, \pi(N-2)\right) \\
\vdots \\
\left(h_{1}, \pi(2), \pi(3), \pi(4), \ldots, h_{2}, \pi(N-2)\right)
\end{array}\right.
$$

for two hub nodes. Note that in the zero-hub node case, all nonhub nodes are included in the logical ring and, thus, without loss of generality, node 1 is taken as the first node (i.e., $\pi(1)=$ 1). For the one-hub node case, by the symmetry of the dual hub architecture, we may assume without loss of generality that the hub node included in the logical topology is $h_{1}$ (i.e., $\pi(1)=h_{1}$ ). Finally, note that there are $N-5$ possible configurations when there are two hub nodes in the logical ring. This includes the case where $h_{2}$ immediately precedes $h_{1}$, which follows by the symmetry of the physical topology. Thus, we have a complete description of all possible ring logical topologies consisting of $N-2$ nodes that must be routed on the dual hub architecture.

We now proceed to demonstrate that when $N$ is even, each of the three configurations (36)-(38) may be routed using the dual hub architecture. First, consider the case of zero hub nodes in the logical topology. Starting at node 1 in the logical ring, a path can be found to the next node via one of the hub nodes. The path to the subsequent node will then go through the other hub node. Thus, consecutive lightpaths in the logical ring alternate between using each of the two hubs as intermediate nodes. Since each hub node can be used as an intermediate node $[(N-2) / 2\rfloor$ times, we can support $N-2$ lightpaths between nodes in the logical topology, as desired. Thus, the dual hub architecture is sufficient to route any logical ring not containing the hub nodes.

Next, we consider the case of a single-hub node in the ring logical topology. Starting with the hub $h_{1}, h_{1}$ has a direct connection to $\pi(1)$, which can connect to $\pi(2)$ through $h_{2}$. Again, we reach the nodes $\pi(i)$ for $i=1, \ldots, N-3$, by alternating between the hub nodes. Following this progression, node $\pi(N-3)$ is reached from hub $h_{1}$. Thus, the last logical link connects node $\pi(N-3)$ to hub $h_{1}$ through nodes $h_{2}$ and $\pi(N-2)$.

Finally, we consider the case where the ring logical topology contains both hub nodes. Denote the two nonhub nodes not included in the logical ring by $\gamma_{1}$ and $\gamma_{2}$. We distinguish between two cases. First, consider the case where no nodes or an even number of nodes separate the hubs. Then, starting with the hub $h_{1}$, traversing the nodes separating $h_{1}$ and $h_{2}$ on the logical ring (or remaining at $h_{1}$ in the case of zero nodes separating the hubs) in the same alternating manner as above implies that we arrive at hub $h_{1}$ rather than $h_{2}$. We then reach node $h_{2}$ by traversing $\gamma_{1}$. Continuing to alternate between the hubs in 
reaching the remaining nodes, we reach the last node in the logical ring $\pi(N-2)$ by passing through hub $h_{1}$. To complete the logical ring, $h_{1}$ must be reached by traversing nodes $h_{2}$ and $\gamma_{2}$. Next, consider the case where an odd number of nodes separate the hubs. Then, again starting with hub $h_{1}$, traversing the nodes separating $h_{1}$ and $h_{2}$ on the logical ring in the same alternating manner as above implies that we arrive at hub $h_{2}$ directly. Continuing to alternate between the remaining nodes, an odd number of nodes remain which implies that there is a direct link back to $h_{1}$ from $\pi(N-2)$.

\section{REFERENCES}

[1] H. Zang, J. P. Jue, and B. Mukherjee, "A review of routing and wavelength assignment approaches for wavelength-routed optical WDM networks," Opt. Networks Mag., pp. 47-60, Jan. 2000.

[2] S. Ramamurthy and B. Mukherjee, "Survivable WDM mesh networks: Part I - Protection," in Proc. INFOCOM, New York, Mar. 1999, pp. 744751 .

[3] $\frac{1}{-}$, "Survivable WDM mesh networks: Part II - Restoration," in Proc. ICC, Vancouver, BC, Canada, June 1999, pp. 2023- 2030.

[4] O. Gerstel, R. Ramaswami, and G. Sasaki, "Fault tolerant multiwavelength optical rings with limited wavelength conversion," in Proc. INFOCOM, Kobe, Japan, Apr. 1997, pp. 507-515.

[5] M. Medard, S. Finn, and R. A. Barry, "WDM loop-back recovery in mesh networks," in Proc. INFOCOM, New York, Mar. 1999, pp. $752-759$.

[6] A. Fumagalli et al., "Survivable networks based on optimal routing and WDM self-healing rings," in Proc. INFOCOM, New York, Mar. 1999, pp. 726-733.

[7] O. Crochat and J. Y. Le Boudec, "Design protection for WDM optical networks," IEEE J. Select. Areas Commun., vol. 16, pp. 1158-1165, Sept. 1998.

[8] B. T. Doshi, S. Dravida, P. Harshavardhana, O. Hauser, and Y. Wang, "Optical network design and restoration," Bell Labs Tech. J., pp. 58-83, Jan.-Mar. 1999.

[9] E. Modiano and A. Narula-Tam, "Designing survivable networks using effective routing and wavelength assignment (RWA)," in Proc. OFC, vol. 2, 2001, pp. TuG5-1-TuG5-3.

[10] - "Survivable routing of logical topologies in WDM networks," in Proc. INFOCOM, Anchorage, AK, Apr. 2001, pp. 348-357.

[11] — "Survivable lightpath routing: A new approach to the design of WDM-based networks," IEEE J. Select. Areas Commun., vol. 20, pp. 800-809, May 2002.

[12] A. Sen, B. Hao, and B. H. Shen, "Survivable routing in WDM networks," in Proc. ISCC, July 2002, pp. 726-731.

[13] A. Narula-Tam and E. Modiano, "Dynamic load balancing for WDM-based packet networks," in Proc. INFOCOM, Tel-Aviv, Israel, Apr. 2000, pp. 1010-1019.

[14] - "Dynamic load balancing in WDM packet networks with and without wavelength constraints," IEEE J. Select. Areas Commun., vol. 18, pp. 1972-1979, Oct. 2000.

[15] C. H. Papadimitriou and K. Steiglitz, Combinatorial Optimization: Algorithms and Complexity. Englewood Cliffs, NJ: Prentice-Hall, 1982.

[16] R. K. Ahuja, T. L. Magnanti, and J. B. Orlin, Network Flows: Theory, Algorithms, and Applications. Englewood Cliffs, NJ: Prentice-Hall, 1993.

[17] E. Lawler, Combinatorial Optimization: Networks and Matroids. New York: Holt, Rinehart and Winston, 1976, p. 122.

[18] ILOG CPLEX 6.5 User's Manual.

[19] R. M. Karp, "On the computational complexity of combinatorial problems," Networks, vol. 5, pp. 45-68, 1975.
[20] M. Kodialam and T. V. Lakshman, "Minimum interference routing with application to MPLS traffic engineering," in Proc. INFOCOM, Tel-Aviv, Israel, Apr. 2000, pp. 884-893.

[21] A. Narula-Tam and E. Modiano, "Network architectures for supporting survivable WDM rings," in Proc. OFC, 2002, pp. 105-107.

[22] A. Narula-Tam, E. Modiano, and A. Brzezinski, "Physical topology design for survivable routing of logical rings in WDM-based networks," in Proc. GLOBECOM, San Francisco, CA, Dec. 2003, pp. 2552-2557.

[23] M. R. Garey and D. S. Johnson, Computers and Intractability: A Guide to the Theory of NP-Completeness. New York, NY: Freeman, 1979.

[24] M. Gondran and M. Minoux, Graphs and Algorithms. New York: Wiley, 1984

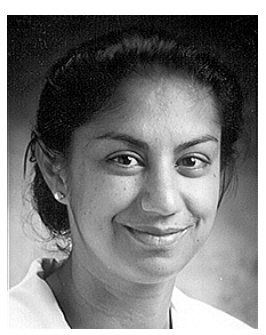

Aradhana Narula-Tam (S'89-M'97) received the B.S.E. degree from the University of Pennsylvania, Philadelphia, in 1990 and the S.M. and $\mathrm{Ph}$.D. degrees from the Massachusetts Institute of Technology (MIT), Cambridge, in 1992 and 1997, respectively, all in electrical engineering.

From 1997 to 1998, she was in the Research and Advanced Development Group, Motorola Information Systems Group, Mansfield, MA. Since 1998, she has been with the Advanced Networking Group, MIT Lincoln Laboratory, Lexington, MA. Her research interests include communication theory, optical networks, and satellite networks.

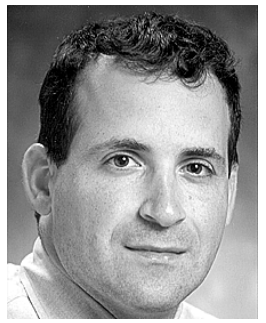

Eytan Modiano (S'90-M'93-SM'00) received the B.S. degree in electrical engineering and computer science from the University of Connecticut, Storrs, in 1986 and the M.S. and Ph.D. degrees from the University of Maryland, College Park, in 1989 and 1992, respectively, both in electrical engineering.

He was a Naval Research Laboratory Fellow from 1987 to 1992 and a National Research Council Postdoctoral Fellow from 1992 to 1993 , while he was conducting research on security and performance issues in distributed network protocols. From 1993 to 1999, he was with the Communications Division, Massachusetts Institute of Technology (MIT) Lincoln Laboratory, Cambridge, where he designed communication protocols for satellite, wireless, and optical networks and was the Project Leader for MIT Lincoln Laboratory's Next-Generation Internet (NGI) Project. He joined the MIT faculty in 1999, where he is presently an Associate Professor in the Department of Aeronautics and Astronautics and the Laboratory for Information and Decision Systems (LIDS). His research is on communication networks and protocols with emphasis on satellite, wireless, and optical networks.

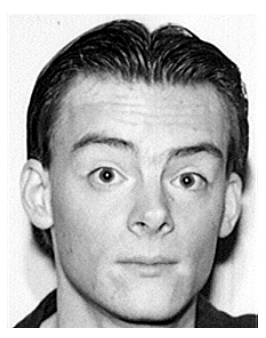

Andrew Brzezinski (S'99) received the B.A.Sc. degree in electrical engineering from the University of Toronto, Toronto, ON, Canada, in 2000 and the M.S. degree in electrical engineering from Stanford University, Stanford, CA, in 2002. He is currently working toward the Ph.D. degree in electrical engineering with the Laboratory for Information and Decision Systems, Massachusetts Institute of Technology (MIT), Cambridge.

His current research interests are in optical communication networks. Additionally, he is interested in information theory, wireless communication, and queueing theory. 